\title{
STUDIES OF STRUCTURE AND DYNAMICS OF SOLID POLYMERS BY ELASTIC AND
} INELASTIC NEUTRON SCATTERING

Erhard W. Fischer

Institut für Physikalische Chemie der Universität Mainz, D - 6500 Mainz, Jakob-Welder-Weg 15 and Sonderforschungsbereich 41 Mainz/ Darmstadt (Federal Republic of Germany)

(The measurements were carried out partly at the Institute Laue Langevin, Grenoble, and partly at the Kernforschungsanlage Jülich).

Abstract - Neutron scattering methods can be applied to solid polymers in order to investigate the structure of the bulk material as well as the nature of the molecular motion. A review of some recently performed studies is presented. Diffusional motion in crystals of chain molecules has been studied by means of quasielastic scattering for the case of $n-\mathrm{C}_{33} \mathrm{H}_{68}$ as an example for the relationship between structure and molecular motion. It can be shown that below the so-called rotational transition the paraffin crystals exhibit two types of molecular motion: a translational jump $\left(\tau_{t}=0.510^{-8} \mathrm{~s}\right)$ and a $180^{\circ}$-rotational jump $\left(\tau_{r}=2.510^{-8} \mathrm{~s}\right)$.

The low frequency vibrations in $n$-alkanes as models of polyethylene have been investigated by means of inelastic incoherent scattering. The longitudinal acoustical mode as well as other lattice vibrations are observed with frequencies, which are in good agreement with the calculations by Kitagawa and Miyazawa.

As examples for structure studies the results of neutron scattering of cellulose fibres "stained" by deuterium and of semicrystalline statistical copolymers are mentioned. Further the problem of chain configuration in crystalline polymers is discussed to some extent. It is shown that the preliminary results obtained in various laboratories do not allow a uniform conclusion, but there is overwhelming evidence that in the case of crystallization from the melt the observed scattering curves are inconsistent with the model of adjacent re-entry and regular folds.

\section{1) INTRODUCTION}

Recently considerable interest has grown in the application of various kinds of neutron scattering methods to polymer science $(1-4)$. These methods can be used for the investigation of the structure of solid polymers and of the configuration of chain molecules as well as for the study of dynamic properties. The growth of this field is mainly due to the development of new techniques applicable to problems in polymer science (e.g. small angle neutron scattering) and further to the improved availability of neutron sources with a sufficiently high flux. 
The scattering of the neutrons from the nuclei of the atoms of the polymer sample is described by the double differential cross section $\partial^{2} \sigma / \partial \Omega \partial E$, which is the probability that an incident neutron (wave vector $\underline{k}$ ) will be scattered in a given direction (wave vector $\underline{k}^{\prime}$ ) with an energy between $E$ and $E+d E$, see Fig.1. That scattering cross section depends on the momentum transfer in the scattering event

$$
\hbar \underline{\underline{Q}}=\hbar\left(\underline{k}-\underline{k}^{\prime}\right)
$$

and on the energy transfer

$$
\Delta E=\hbar \omega=\frac{\hbar^{2}}{2 m}\left(k^{2}-k^{\prime 2}\right)
$$

where $m$ is the mass of the neutron. (Sometimes $k$ is used instead of Q.)

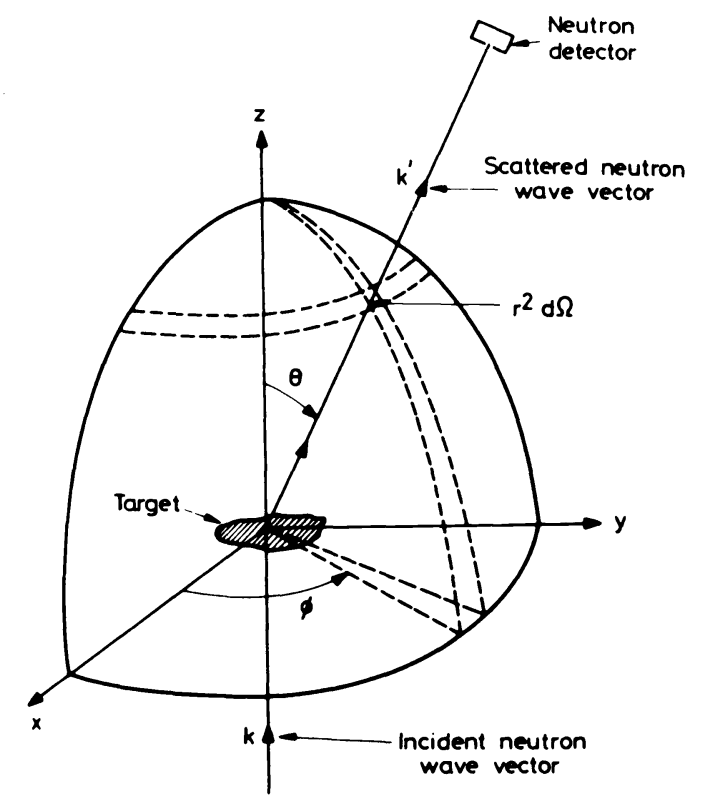

Fig. 1: Incident and scattered neutron waves described by wave vectors $\underline{k}$ and $\underline{k}^{\prime}$, resprectively (4). $d \Omega$ is the fractional solid angle.

The theory of thermal neutron scattering is well developed $(5-8)$. However, there are two important features which we need to know in order to understand the applications to polymers. Because of the spin of the neutron, a nucleus with spin I $\neq 0$ scatters with different scattering lengths $b$ depending on the total momentum $I \pm \frac{1}{2}$. Consequently the scattering cross section $\sigma$ consists of two components: a coherent $\sigma_{\text {coh }}$ and an incoherent $\sigma_{\text {incoh }}$ part, even if the sample contains only one kind of nuclei. The coherent scattering is associated with interference effects. It can be used for structure investigations. Fortunately there is a large difference between the coherent scattering amplitudes of hydrogen and deuterium, which provides the base for the application of small angle neutron scattering to the investigation of chain configurations in the bulk state.

In addition one has to distinguish between an elastic part of the scattering, for which $\Delta \mathrm{E}=\hbar \omega=0$, and an inelastic part with positive or negative energy transfer. The inelastic scattering allows us to study the dynamics of polymer systems. Regarding the ranges of momentum and energy transfer for various kinds of scattering experiments (i.e. X-ray, light, 
electron scattering) it turns out that only with neutrons both $\underline{Q}$ and $\Delta E$ are simultaneously of the right order of magnitude for studying the motion on a molecular scale.

According to these effects neutron scattering experiments can be divided into 4 groups as specified in Table 1. Some possible applications to problems of polymer physics are enumerated, al though most of them have not been studied extensively so far.

Table 1: Information that can be derived from neutron scattering experiments on solid polymers.

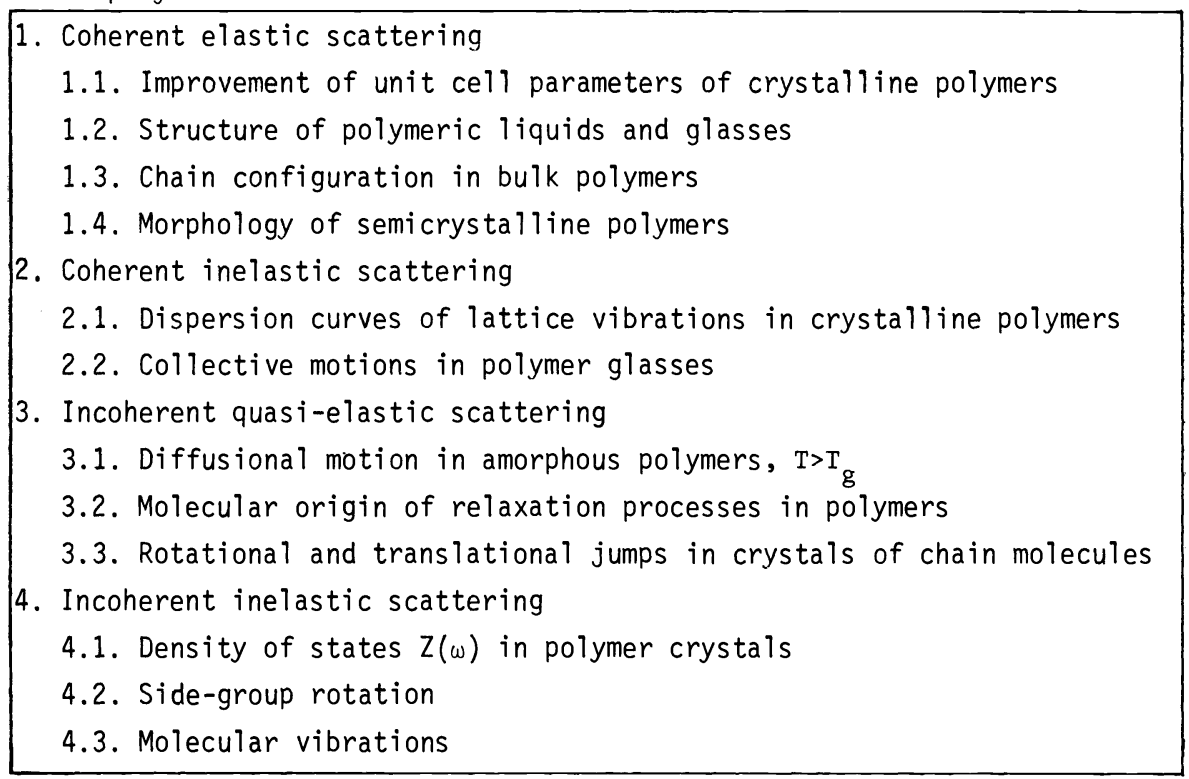

In the following I shall first present two examples of dynamic studies carried out in our laboratory (belonging to sections 3.3 and 4.1 of Table 1) and thereafter concern myself with the elastic coherent scattering as applied to problems of morphology and chain configuration in semicrystalline polymers (sections 1.3 and 1.4).

\section{2) DIFFUSIONAL MOTIONS IN CRYSTALS OF CHAIN MOLECULES}

From mechanical, dielectrical or NMR relaxation experiments it is well known that chain molecules may exhibit a considerable degree of molecular motion even in the crystalline state. If one wants to study the non-collective motion of individual molecular groups by means of neutron scattering one has to measure the incoherent differential cross section, because the coherent part of the inelastic scattering is determined by the collective motions of the system (phonons). The neutrons will gain or lose small amounts of energy and therefore one observes a quasi-elastic broadening of the elastic peak. The differential cross section may be written as

$$
\frac{\partial^{2} \sigma_{i n c}}{\partial \Omega \partial \mathrm{E}} \sim \frac{N \sigma_{i n c}}{4 \pi \hbar} \frac{k^{\prime}}{k} \quad S_{i}(\underline{Q}, \omega)
$$


where $\mathrm{N} \sigma$ inc is the cross section of the sample containing $\mathrm{N}$ protons. (Eq. (3) takes into account that $\sigma_{i n c}$ for protons is much larger than for all other nuclei.) The important quantity in eq. (3) is the so-called scattering law or dynamical structure factor $S_{j}(\underline{Q}, \omega)$ which can be shown ( 9 ) to be equal to the Fourier transform of the self correlation function $G_{S}(\underline{r}, t)$ :

$$
S_{i}(\underline{Q}, \omega)=\frac{1}{2 \pi} \int^{\prime} \exp [i(\underline{Q} \underline{r}-\omega t)] \cdot G_{s} \underline{(\underline{r}, t)} d t d \underline{r}
$$

In the classical limit the space-time correlation function $G_{s}\left(\underline{r}-\underline{r}^{\prime}, t-t^{\prime}\right)$ gives the ensemble averaged probability that the scattering nucleus is found at time $t$ at $\underline{r}$, if it has been at $\underline{r}^{\prime}$ at an earlier time $t^{\prime}$. Evidently any motion of the particle can be described by this function.

These principles have been applied (10) to the molecular motions of $n$-alkanes. As has been shown previously (11) a highly purified sample of n-tritriacontane $\left(\mathrm{C}_{33} \mathrm{H}_{68}\right)$ exhibits four stable crystal modifications before melting $\left(T_{m}=71.8^{\circ} \mathrm{C}\right)$, see Fig.2. The phase transitions observed at $54.5^{\circ} \mathrm{C}, 65.5^{\circ} \mathrm{C}$ and $68^{\circ} \mathrm{C}$ are accompanied by different types of disorder and the onset of specific types of molecular motion. By a combination of structure investigations, wide line NMR experiments, and infrared measurements it was possible to deduce special motional mechanisms in relation to the various structures. Figure 3 demonstrates schematically the conclusions of the earlier work.

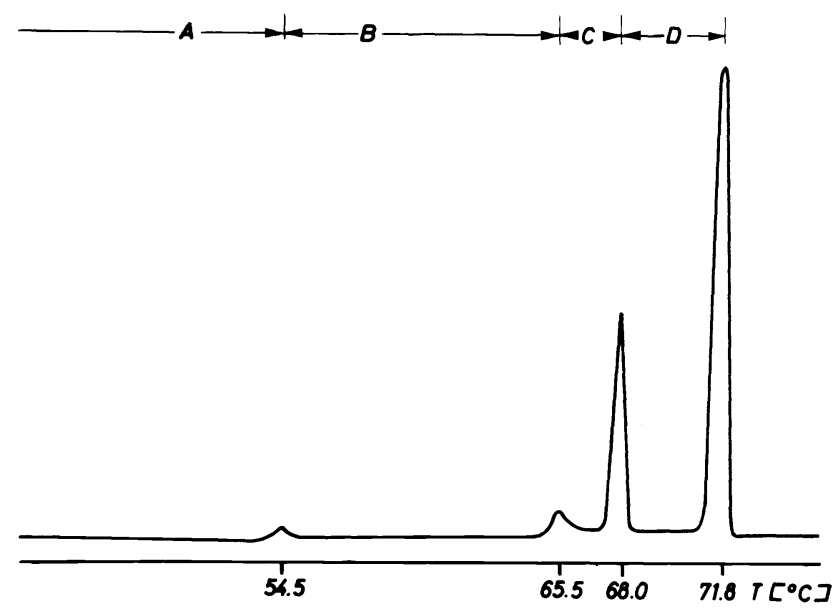

Fig. 2: DSC-thermogram of n-tritriacontane $\left(\mathrm{C}_{33} \mathrm{H}_{68}\right)$ showing four stable crystal modifications, $A, B, C$ and $D$ before melting 


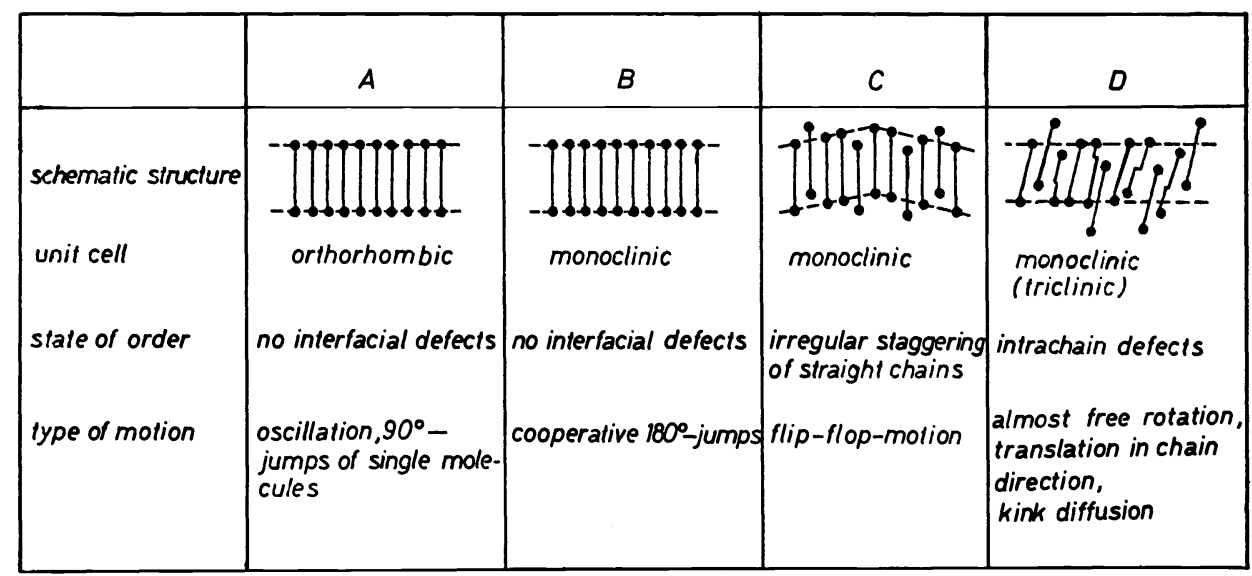

Fig. 3: States of order, types of motion and schematic diagrams of the structures of the four modifications of $n-\mathrm{C}_{33} \mathrm{H}_{68}$ (11).

We are now particularley interested in the modification $C$, because NMR studies of polyethylene showed that the molecular motion beyond the well-known a-relaxation of the polymer is strongly related to that of the modification $C$ in alkanes. It was assumed that molecular motion in $C$ is characterized by a superposition of $180^{\circ}$ rotational jumps of the whole chain and a simultaneous shift in chain direction by half a repeating distance [so-called flip-flop jumps $(12,13)$, see Fig. $4 . j$ It seemed interesting to prove this assumption by means of quasielastic neutron scattering.

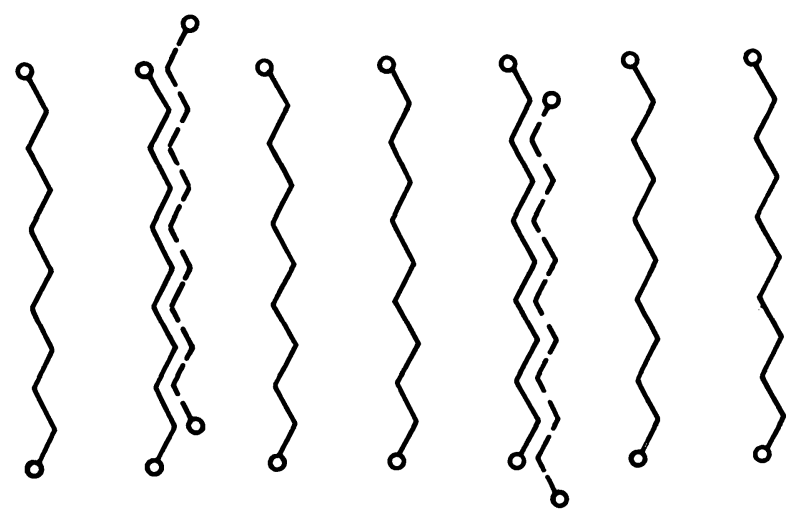

Fig. 4: Flip-flop screw jumps in a paraffin crystal $(11-13)$. 
An uniaxially oriented sample of $\mathrm{C}_{33} \mathrm{H}_{68}$ crystals was obtained by precipitation from solution, filtering and pressing. The scattering experiments were performed using a back scattering spectrometer (14), the principle of which is shown in Fig. 5. The scattering vector $\underline{Q}$ was oriented either mainly parallel or perpendicular to the chain axis.

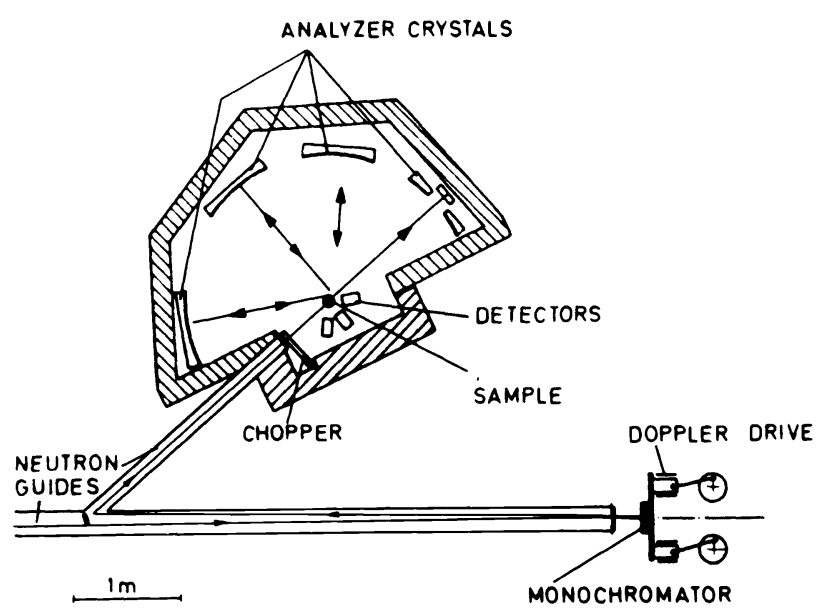

Fig. 5: High resolution backscattering spectrometer for quasielastic neutron scattering experiments (14).
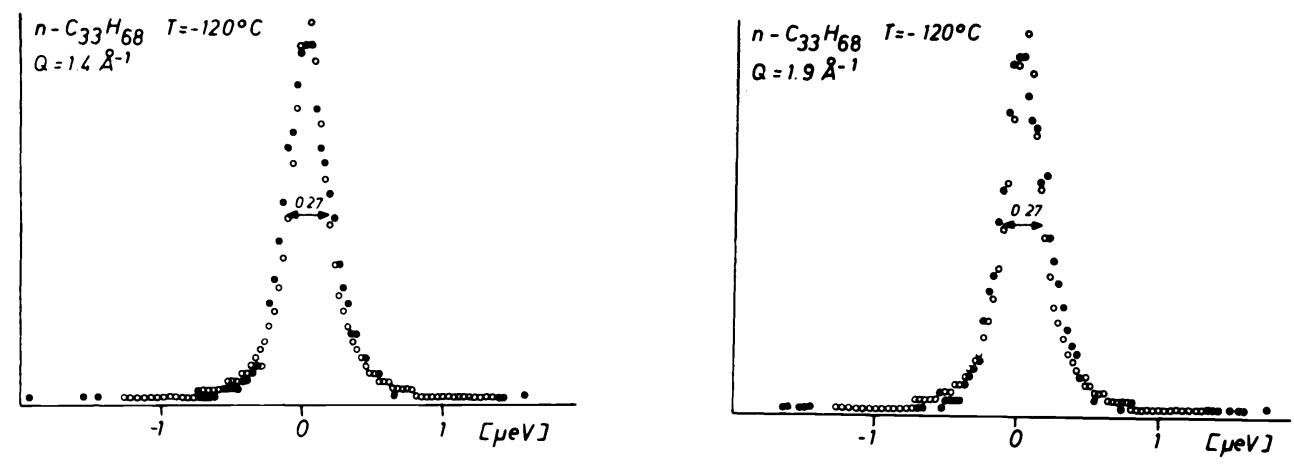

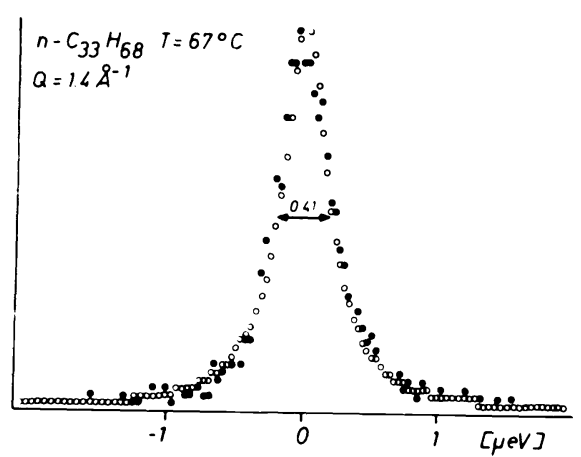

(a)

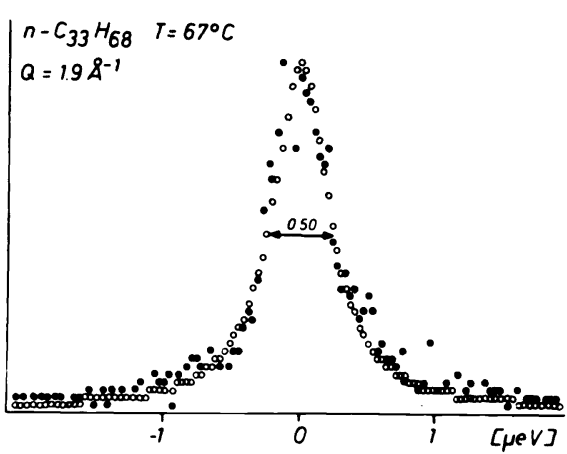

(b)

Fig. 6: Quasielastic neutron scattering of uniaxially oriented $n-C_{33} H_{68}$ crystals (10). (a) Q perpendicular to the chain axes, (b) Q parallel to the chain axes, o experimental spectrum, fitted Lorentzian curve. 
As examples, Fig. 6 shows some quasielastic spectra. For comparison the spectra measured at $-120^{\circ} \mathrm{C}$, are also shown. At this temperature the molecular motion is frozen in, i.e. these curves are determined by the instrumental resolution. From the spectra obtained at various scattering vectors $\underline{Q}$ the nature of the molecular motion could be deduced by comparing the measured spectra with the dynamic structure factors which have been calculated for various types of motion (10). The results are summarized in Table 2 and indicate that the results of the quasi-elastic neutron scattering experiments do not agree with the assumption of a flipflop motion, since the time constants for the rotational jump and the translational jump are different. It will be interesting to discuss this observation in terms of potential energy calculations for the n-alkane or polyethylene lattices similar to those carried out by Mc Cullough (15).

Table 2: Nature of chain motion in the modifications $C$ and $D$ of $n-C_{33}{ }^{H} 68$.

\begin{tabular}{|c|c|c|}
\hline modification & X-ray, NMR (11) & $\begin{array}{l}\text { quasielastic neutron } \\
\text { scattering (10) }\end{array}$ \\
\hline C & $\begin{array}{l}\text { flip-flop screw jumps } \\
\text { Identical } \tau \text { values have } \\
\text { to be expected for the } \\
\text { motional components } \\
\text { parallel and perpendicular } \\
\text { to the chain axes }\end{array}$ & $\begin{array}{l}180^{\circ} \text { rotational jumps } \\
\left(\tau_{\mathrm{r}}=2.5 \cdot 10^{-8} \mathrm{~s}\right) \\
\text { and trans lational jumps } \\
\left(\tau_{t}=0.5 \cdot 10^{-8} \mathrm{~s}\right) \\
\text { independent from each other }\end{array}$ \\
\hline D & $\begin{array}{l}\text { almost free rotation, } \\
\text { motion of whole chains in } \\
\text { chain direction and kink } \\
\text { diffusion }\end{array}$ & $\begin{array}{l}\text { independent rotational and } \\
\text { translational diffusion } \\
\left(\tau_{\mathrm{D}} \approx 10^{-10} \mathrm{~S}\right)\end{array}$ \\
\hline
\end{tabular}

\section{3) LOW FREQUENCY VIBRATIONS OF AN N-ALKANE AS A MODEL OF POLYETHYLENE}

Besides the diffusional type of motion, the molecular motion which is due to vibrations can also be studied by means of neutron scattering. In contrast to infrared and Raman spectra there are no selection rules and consequently all the vibrations of molecules contribute to inelastic scattering of thermal neutrons. In the case of a molecular crystal only vibrations with the wave vector $\underline{k}=0$ can be observed by infrared and Raman techniques, whereas all crystal vibrations contribute to the neutron scattering cross section.

Inelastic coherent scattering is due to the collective motion of atoms or molecular groups and thus allows us to analyse the dispersion relation $\omega(\underline{K})$ between the vibrational frequencies and the wave vector $\underline{k}$ of the phonons. This is an important method for the study of crystal dynamics. It has been applied to polymers $(3,16)$ and interesting results have been obtained. Difficulties arise from the lack of macroscopic single crystals and from the requirement to use deuterated polymers because of the low coherent cross section of hydrogen $\left(1.8 \cdot 10^{-24} \mathrm{~cm}^{2}\right)$. 
The incoherent scattering cross section of the proton is much larger $\left(79.7 \cdot 10^{-24} \mathrm{~cm}^{2}\right)$ and therefore the incoherent inelastic scattering is predominant in normal polymer crystals. Its analysis yields the frequency distribution primarily of the protons weighted with their vibrational amplitudes:

$$
\begin{aligned}
& \frac{\partial^{2} \sigma_{\text {inc }}}{\partial \Omega \partial E} \sim \frac{k^{\prime}}{k} b_{\text {inc }}^{2} \frac{Q^{2}\left\langle u^{2}\right\rangle}{2 M} \frac{z(\omega)}{\omega} \\
& \left\langle u^{2}\right\rangle=\text { mean square amplitude of vibration } \\
& Z(\omega)=\text { density of states of the vibrational spectrum }
\end{aligned}
$$

As an example of the application of this method I want to describe a study of the low frequency vibrations of normal paraffin which was investigated as a model of crystalline polyethylene (17).

The normal vibrations of orthorhombic polyethylene have been calculated by Kitagawa and Miyazawa (16) and by other authors (18). Some of the theoretical dispersion curves are plotted in Fig. 7 (with regard to the mode symbols we refer to the original paper). According to eq. (5) the density of states $Z(\omega)$ is pictured by the incoherent scattering cross section, i.e. only the cut-offs of the dispersion curves can be observed as peaks or shoulders. If one uses crystals of $n$-alkanes, however, the phase differences along the chain axes are no longer continuously distributed, and consequently series of discrete points on the dispersion curves are obtained. Scattering peaks corresponding to these points are observed, i.e. the progression bands are made visible by the inelastic neutron scattering experiment.

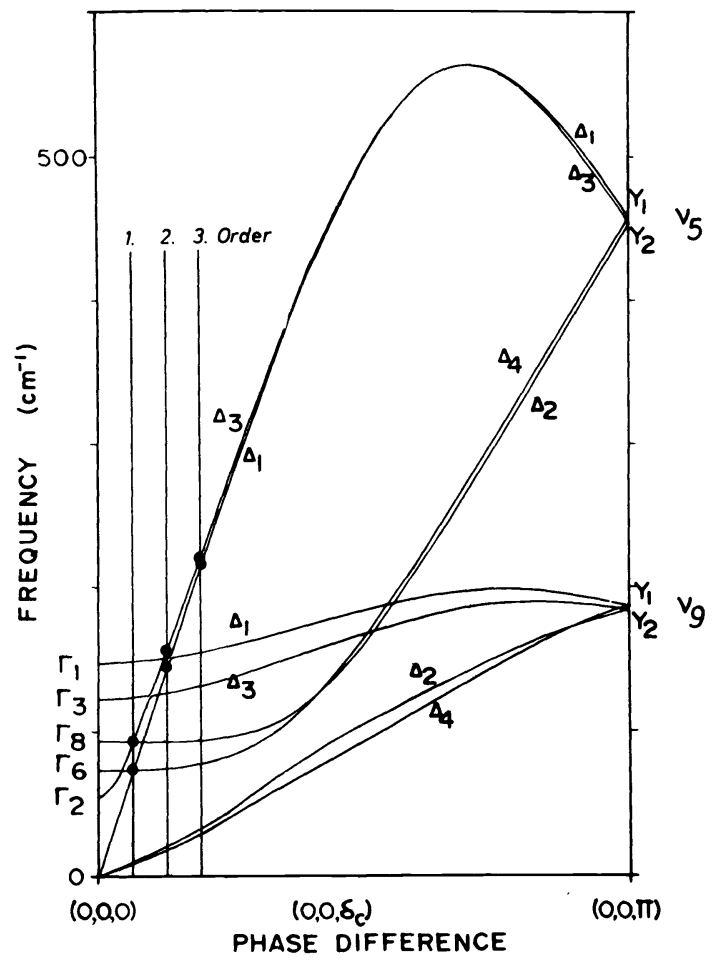

Fig. 7: Frequency dispersion curves of orthorhombic polyethylene crystals for phase differences $\delta$ along the chain axis Kitagawa and Miyazawa (16).

We studied crystals of $n$-dotriacontane $\left(\mathrm{C}_{32} \mathrm{H}_{66}\right)$ grown from dilute solution and uniaxially oriented by slow filtration and pressing at room temperature. The measurements were carried out with a triple-axis spectrometer or with a time-of-flight spectrometer (see Fig. 8). The sample was oriented in such a way, that the scattering vector $\underline{Q}$ was predominantly parallel or perpendicular to the chain axis. 


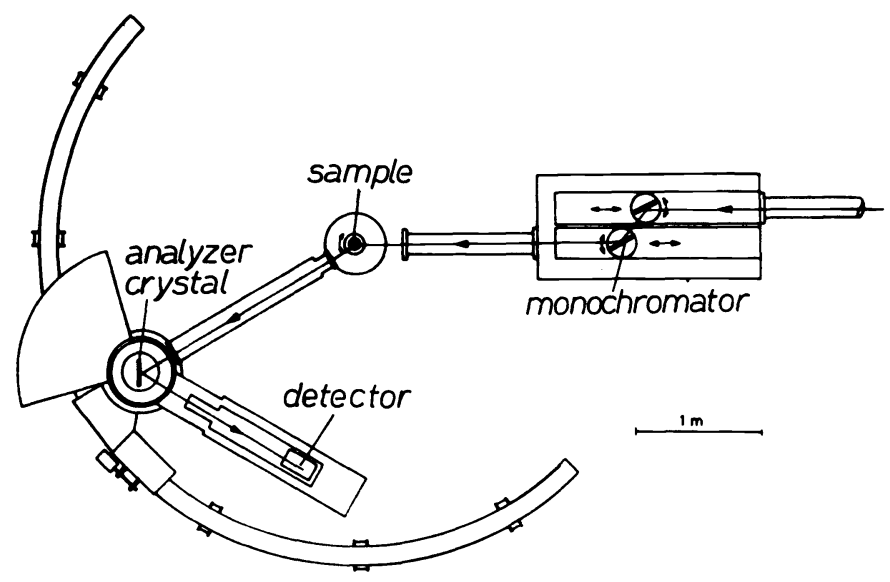

(a)

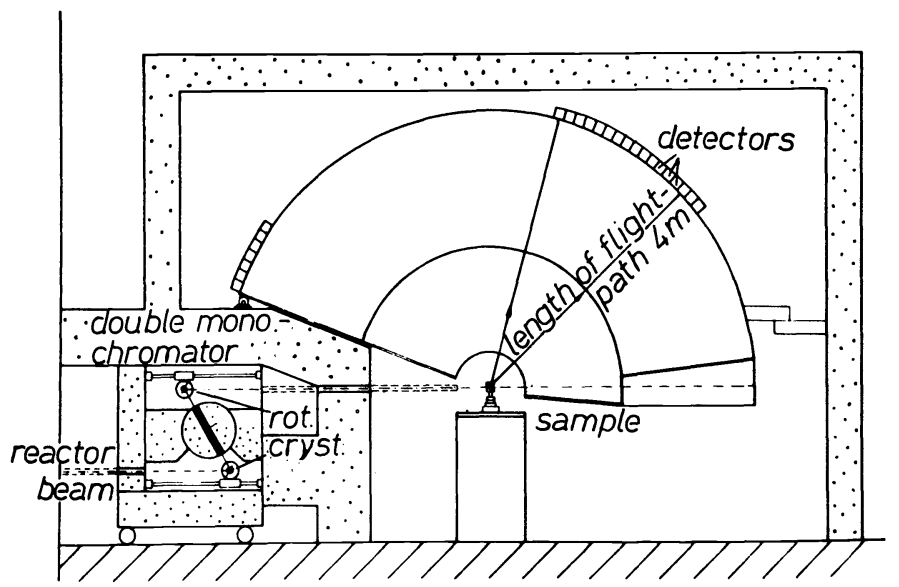

(b)

Fig. 8: Schematic drawings of the equipment used for measuring incoherent inelastic neutron scattering.

(a) Triple-axis spectrometer (Kernforschungsanlage Jülich)

(b) Time-of-flight spectrometer (Institute von Laue-Langevin, Grenoble)

As an example, Fig. 9 shows the triple-axis energy gain spectrum measured at $200 \mathrm{~K}$ with $\underline{Q}$ parallel to the chain axis. The peak at $40 \mathrm{~cm}^{-1}$ can be assigned to the antiparallel translatory vibration mode along the chain axis, because it is not found in the spectrum measured for $\underline{Q}$ perpendicular to the $c$ - direction. This mode cannot be observed by optical spectroscopy because of the selection rules, but it has been predicted theoretically (16). The second peak $B$ at about $80 \mathrm{~cm}^{-1}$ will be discussed in connection with Fig. 10 (it is rather weak in the triple-axis spectrum due to a deep dip of the analyser reflectivity near this energy range). The peaks $C, D$, and $E$ as well as those obtained for $\underline{Q}$ perpendicular to the $c$ - axis can be explained in complete accordance with the theoretical dispersion curves of Fig. 7 (for details we refer to the original paper). 


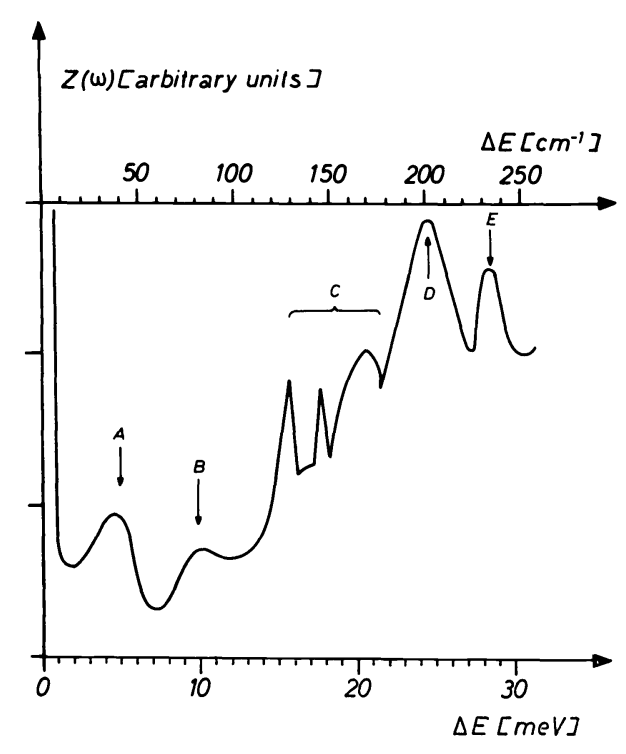

Fig. 9: Density of states of uniaxially oriented crystal mats of $\mathrm{n}-\mathrm{C}_{32} \mathrm{H}_{66}$ versus energy transfer (17). The spectrum was measured with a triple-axis spectrometer at $200 \mathrm{~K}$. Scattering vector $\underline{Q}$ parallel to chain axis.

A spectrum obtained by the time-of-flight method is shown in Fig. 10. The measurements were carried out at $5 \mathrm{~K}$ with $\underline{Q}$ parallel to $\mathrm{c}$. The optically inactive antiparallel translatory mode A appears as a shoulder because of the strong and rather broad elastic peak. The position can be estimated to be about $50 \mathrm{~cm}^{-1}$ in agreement with the theoretical values (16) calculated for $100 \mathrm{~K}$. The difference against the energy gain found in Fig. $9\left(40 \mathrm{~cm}^{-1}\right)$ is supposed to be caused by the variation of the temperature of measurements. The intermolecular forces decrease with increasing temperature and therefore this mode is very sensitive to thermal expansion.

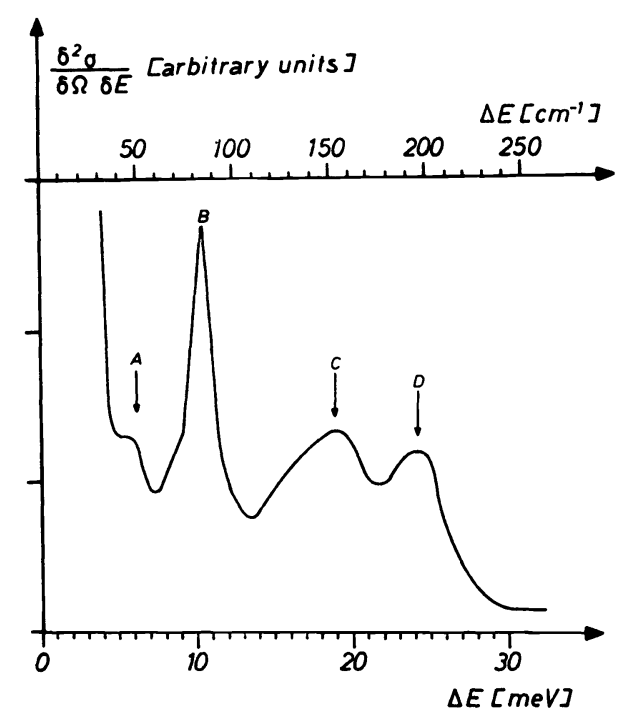

Fig. 10: Time-of-flight spectrum of $n-\mathrm{C}_{32} \mathrm{H}_{66}$ measured at 5 K. $\underline{Q}$ parallel to chain axis (17). Uncorrected data. 
The second strong peak at $B$ with a maximum at $83 \mathrm{~cm}^{-1}$ is the first order progressive band of the skeleton CCC bending vibration mode. The energy gain of this band (10.3 meV) is somewhat larger than the value of $74.7 \mathrm{~cm}^{-1}$ which we found in the raman spectrum of the same sample and which agrees well with the value reported by Schaufele and Shimanouchi (19). The difference is believed to be related to the selection rules. Raman scattering gives the first order of the longitudinal acoustical mode, i.e. the progressive band of the $\Delta_{1}$ branch in Fig. 7. The neutron spectrum, however, will show some averaging over other modes, too. Judging from the dispersion curves of Fig. 7 the density of states has maxima at $\Delta_{1}=73 \mathrm{~cm}^{-1}$, and $\Delta_{3}=93 \mathrm{~cm}^{-1}$. The observed maximum is considered to be intermediate between the $\Delta_{1}$ and $\Delta_{3}$ branches.

The broad peak at $C$ is due to the second order of progressive bands of the CCC bending vibrations; it covers a range between 130 and $170 \mathrm{~cm}^{-1}$. The reason for this broadening is probably the complex nature of the dispersion relations for the phase difference 'c around thiat belonging to the second order progressive bands.

These few examples may suffice to show how measurements of the differential cross section of the incoherent neutron scattering can be analysed in terms of lattice vibrations. It turned out that the neutron spectra of $n-\mathrm{C}_{32} \mathrm{H}_{66}$ can be explained statisfactorily on the basis of the theoretical results obtained by Kitagawa et al. (16). In particular, we were able to verify the expected antiparallel translational mode at $50 \mathrm{~cm}^{-1}$, which is inactive in infrared absorption or Raman scattering. We were also able to demonstrate the existence of progression bands in the neutron scattering spectra of paraffins.

\section{4) MORPHOLOGICAL STUDIES OF SEMICRYSTALLINE POLYMERS BY MEANS OF NEUTRON SMALL ANGLE SCATTERING}

The coherent elastic scattering of neutrons can be applied to solve structure problems of semicrystalline polymers (see Table 1). The advantage of small angle neutron scattering (SANS) compared with $X$-ray techniques (SAXS) consists in the possibility to improve the scattering contrast by tagging certain molecular groups. A good example is the "staining" of the amorphous regions of cellulose fibres (20) by means of the hydrogen-deuterium exchange of the hydroxy - groups. The accessibility of these groups depends on the degree of chain order in the sense that the reaction takes place preferentially in the disordered regions. By treatment with heavy water vapor long spacing reflections $(L=165 \AA)$ are created which are not present for the untreated sample or in the small angle X-ray pattern (see Fig. 11). The existence of these intensity maxima reveals that the degree of order along the cellulose fibre axis changes almost periodically.

Another example is the labelling of copolymer units in semicrystalline samples of random copolymers. The description of the physical properties of such systems requires the knowledge of the distribution of the comonomer units among the crystalline and amorphous phases. By comparing the scattering power for $X$-rays and neutrons this distribution can be deduced, if the comonomer units are labelled by isotopes. Figure 12 shows neutron scattering curves of random poly(oxymethylenedioxolane) with a dioxolane content of $2.5 \%$. The intensity of the 
long spacing reflection depends on the morphological structure and on the distribution of the comonomer units as is indicated by the different scattering behaviour of the tagged and untagged samples. A complete analysis reveals that the comonomer units are not preferentially excluded from the crystal lattice, but that there is a trend towards an equal distribution among the crystalline and amorphous phases (21).

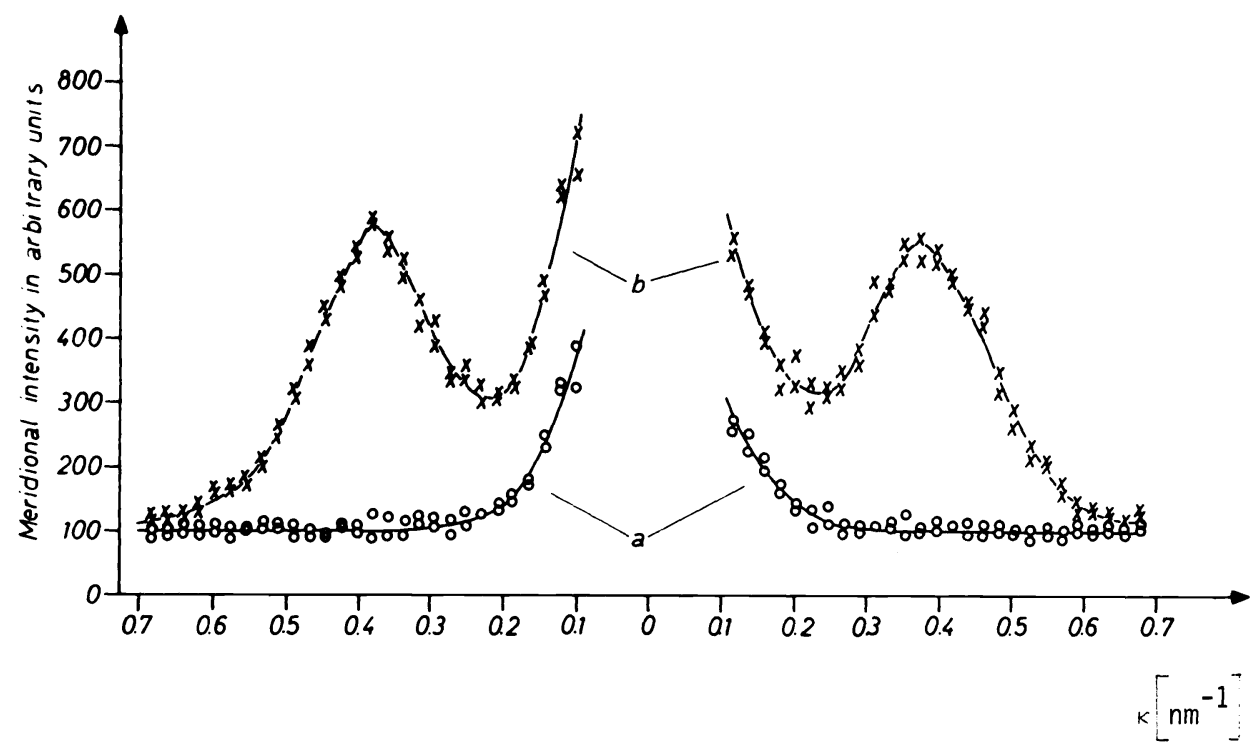

Fig. 11: Meridional small angle neutron scattering curves of regenerated cellulose (20). a) Untreated, b) after selective hydrogen-deuterium exchange by $5 \mathrm{~h}$ treatment with heavy water vapor $\left(\kappa=\frac{4 \pi}{\lambda} \sin \theta / 2\right)$.

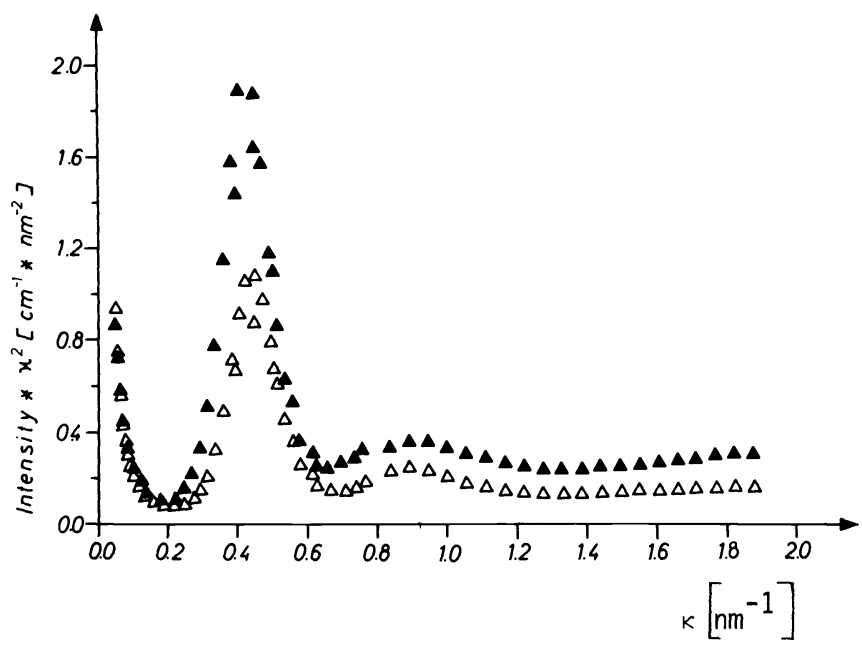

Fig. 12: Neutron small angle scattering curves of poly(oxymethylenedioxolane) copolymers (21). $\triangle$ Fully deuterated, $\Delta$ dioxolane comonomer units protonated. 


\section{5) CHAIN CONFIGURATION IN SEMICRYSTALLINE POLYMERS}

The coherent elastic neutron scattering also allows an investigation of the configuration of molecules in the bulk polymer (see Tab. 1), if the necessary scattering contrast is introduced by dissolving deuterated molecules in an undeuterated matrix or vice versa. The scattering cross section $d \sigma / d \Omega$ is proportional to

$$
\left(a_{1}-a_{2}\right)^{2}\left\langle\left|\sum_{j} e^{i \underline{Q} \underline{r}_{j}}\right|^{2}\right\rangle
$$

where $a_{1}$ and $a_{2}$ are the scattering lenghts per monomer unit of the matrix and of the dissolved chain, respectively (for example: $\mathrm{a}_{\mathrm{CH}_{2}}=-0.07 \cdot 10^{-12} \mathrm{~cm},{ }^{a} \mathrm{CD}_{2}=2.01 \cdot 10^{-12} \mathrm{~cm}$ ).

The method proved to be useful in studying amorphous glassy polymers $(2,22,23)$ and polymer melts $(24,25)$ as well as for the investigation of polymer mixtures, concentrated solutions and polymer networks. It can also be applied to the study of crystalline polymers and the following part of the paper is concerned with a review of the present results.

There has been a long-time controversy about the chain configuration in the semicrystalline state. It is well established that the crystalline lamellae are covered by amorphous surface layers, but various models have been proposed to explain this observation. Figure 13 shows two of such models and demonstrates the well-known problems of chain folding, switchboard structure and adjacent reentry. It is hoped that these questions can be solved by the application of elastic coherent neutron small and wide angle scattering and that from the results conclusions can be drawn with regard to the properties of such systems and to the mechanism of the crystallization process.
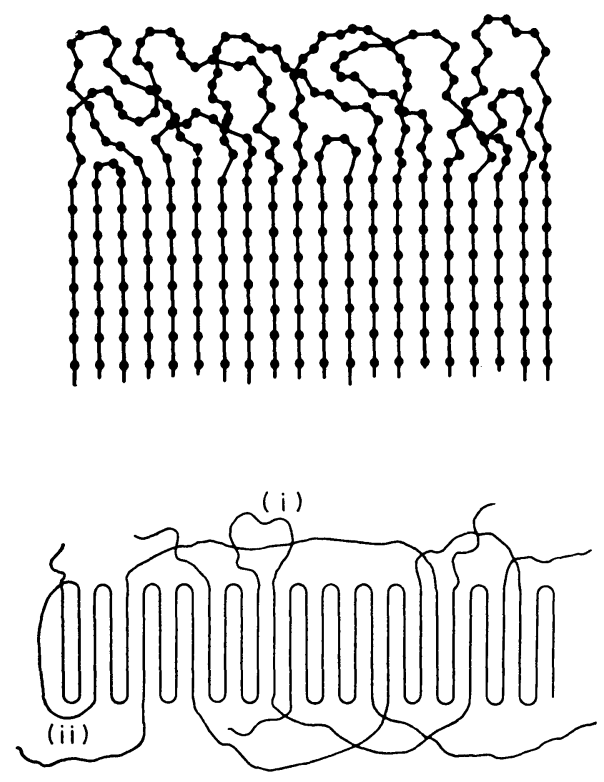

a)

Fig. 13: Two models for the structure of the amorphous surface layers on top of the crystalline lamellae.

a) Non-adjacent re-entry along a net plane (31), a two-dimensional modification of Flory's "switchboard" model (30).

b) Composite fold surface consisting of sharp folds between adjacent re-entrants, loose loops and cilia (32). 
The study of semicrystalline polymers turned out to be more difficult than the investigation of the amorphous state because of experimental obstacles. Measurements of the apparent molecular weight from zero-angle scattering indicated that in some cases - especially with polyethylene - a molecular clustering of the tagged molecules occured during crystallization (25). Another difficulty arises from the background scattering because of microvoids which are often present in samples which were crystallized from solution. Moreover the structure factor of the molecular scattering has to be separated from that caused by the crystalline-amorphous morphology of the sample. These difficulties are responsible for the fact that some of the results reported can hardly be used to draw any conclusions.

It is convenient to divide the studied range of momentum transfer $\underline{Q}$ into three parts:

i) The molecular scattering ( $Q R<1, R=$ radius of gyration) is caused by the overall mass distribution of the polymer chain and yields valuable information about the "dilution" of a single chain in the crystalline state. Further the dependence of the radius of gyration $\left\langle s^{2}\right\rangle^{1 / 2}$ on the molecular weight can be utilized for accepting or refusing certain models. $i i)$ In the submolecular range $(Q R>1)$ certain features of the molecular configuration can be evaluated from the shape of the scattering curve; for example the organization of the crystalline sequences along planes or in lamellae.

iii) The wide angle scattering studies $(Q d \approx 2 \pi), d=$ net plan distance) allow to investigate the distribution of the deuterated stems along certain planes, and it is believed that the problem of adjacent reentry can be studied most conclusively in this scattering range. The radius of gyration is conventionally obtained by plotting $\left(d_{\sigma} / \mathrm{d} \Omega\right)^{-1}$ versus $Q^{2}$. Figure 14 shows such a plot for poly(ethylene oxide) in the molten and crystalline states (26). Surprisingly small changes of the scattering curves are caused by the crystallization. Accordingly the radii of gyration are only slightly affected by the crystallization process. Some of the values so far obtained are presented in Table 3. In the case of isotactic polypropylene (27), where the molecular weight dependence was studied, is was found that the radi $i$ of gyration obey the $M_{w}{ }^{1 / 2}$ law also in the crystalline state. In addition it was shown that $\left\langle s^{2}\right\rangle^{1 / 2}$ does not depend on crystallization or annealing conditions although a drastic change of the long spacing $(120-250 \mathrm{~A})$ was achieved. 


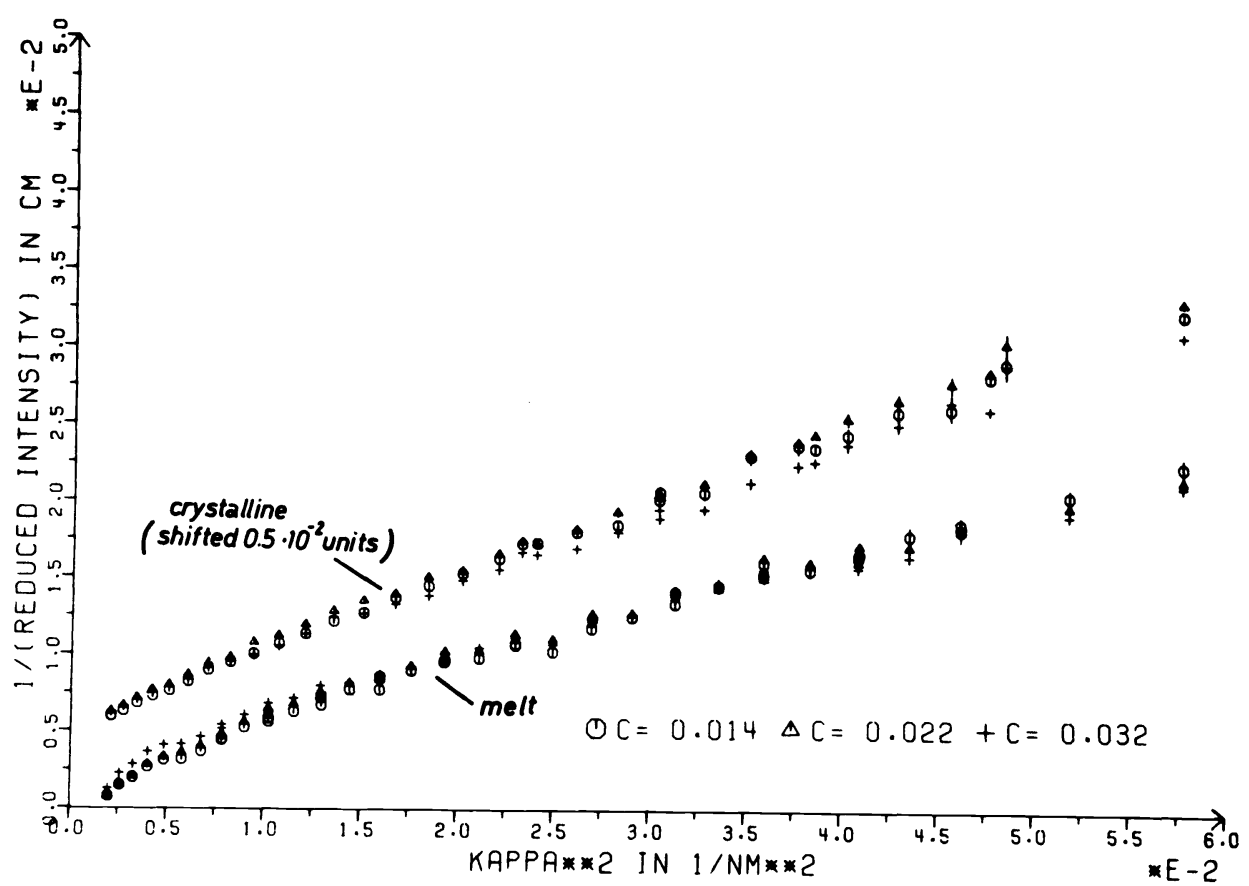

Fig. 14: Comparison of scattering curves of molten and crystallized poly(ethylene oxide) (26) in the range $0.005<k<0.025 \mathrm{~A}^{-1}$. The reciprocal reduced intensity $(d \sigma / d \Omega)^{-1}$ is plotted versus $k^{2}$ (in $10^{-2} \mathrm{~nm}^{-2}$ ). The symbols refer to different concentrations.

Table 3: Comparison between the radii of gyration (expressed by $\left\langle s^{2}\right\rangle^{1 / 2} / M_{W}{ }^{1 / 2}$ ) in the melt and in the semicrystalline state.

\begin{tabular}{|c|c|c|c|c|}
\hline Polymer & $\bar{M}_{W} \cdot 10^{-3}$ & 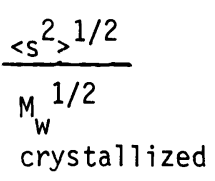 & $\underbrace{\left[\AA /(g / \text { Mol })^{1 / 2}\right]}_{\text {melt }}$ & Ref. \\
\hline $\begin{array}{l}\text { Polyethylene, } \\
\text { quenched }\end{array}$ & $\begin{array}{l}140 \\
\left(M_{w} / M_{n}=2.1\right)\end{array}$ & $\begin{array}{l}0.46 \pm 0.05 \\
0.45\end{array}$ & $\begin{array}{l}0.46 \pm 0.05 \\
0.45\end{array}$ & $\begin{array}{l}(25) \\
(24)\end{array}$ \\
\hline $\begin{array}{l}\text { Polyethylene oxide, } \\
\text { crystallized by } \\
\text { slowly cooling }\end{array}$ & $\begin{array}{l}150 \\
\left(M_{W} / M_{n} \text { large }\right)\end{array}$ & 0.52 & $\begin{array}{l}0.46 \\
0.42\end{array}$ & $\begin{array}{l}(26) \\
(29)\end{array}$ \\
\hline $\begin{array}{l}\text { Isotactic polypro- } \\
\text { pylene, isothermal- } \\
\text { ly crystallized }\end{array}$ & $\begin{array}{l}340 \\
\left(M_{w} / M_{n}=2\right) \\
1540 \\
\left(M_{w} / M_{n}=1.5\right)\end{array}$ & $\begin{array}{l}0.37 \\
0.38\end{array}$ & 0.34 & $(36)$ \\
\hline
\end{tabular}


It is obvious that these observations are difficult to explain on the basis of a model assuming regular chain folding along a net plane (sheet - structure). The fold length is fixed by the matrix and very special and rather artifical assumptions on the widths of the sheets and on their spatial distribution must be introduced in order to arrive at the observed radius of gyration $(25,27)$.

The most simple explanation for the approximate invariance of $\left\langle s^{2}\right\rangle^{1 / 2}$ may be based on a model, which we call "Erstarrungsmodel1" (solidification model). In this model it is assumed that crystallization occurs by straightening of the coil without a long range diffusion process. Figure 15 shows schematically how a part of a coil inserts into the growing lamellae creating a switchboard type structure $(30,33)$ of the amorphous interlamellar layers. Such a model has been treated theoretically by Zachmann et al. (34) and the end-toend distribution of the loops has been calculated. Evidently, the space occupied by a single coiled molecule is not changed appreciably by such a crystallization process and the radius of gyration will therefore remain constant.

Besides the radius of gyration, which describes the size of the overall mass distribution of a single molecule in the crystalline state, additional information about the configuration of the molecules can be obtained from measurements at larger scattering vectors $\underline{Q}$. The question arises whether the model of Fig. 15 is consistent with the observed neutron scattering curves. Conventionally the form factor $P(Q)$ of the molecule is studied by plotting $I(Q) Q^{2}$ vs. $Q$ (Kratky - plot) and by comparing the shape of this curve in the range $0.02 \leq Q \leq 0.2 \AA^{-1}$ with theoretical predictions for various models of the configuration of an isolated chain in the semicrystalline polymer. Again various experimental difficulties arise, the most important being background scattering, whereas the clustering problem plays no large role in this range of $Q$.
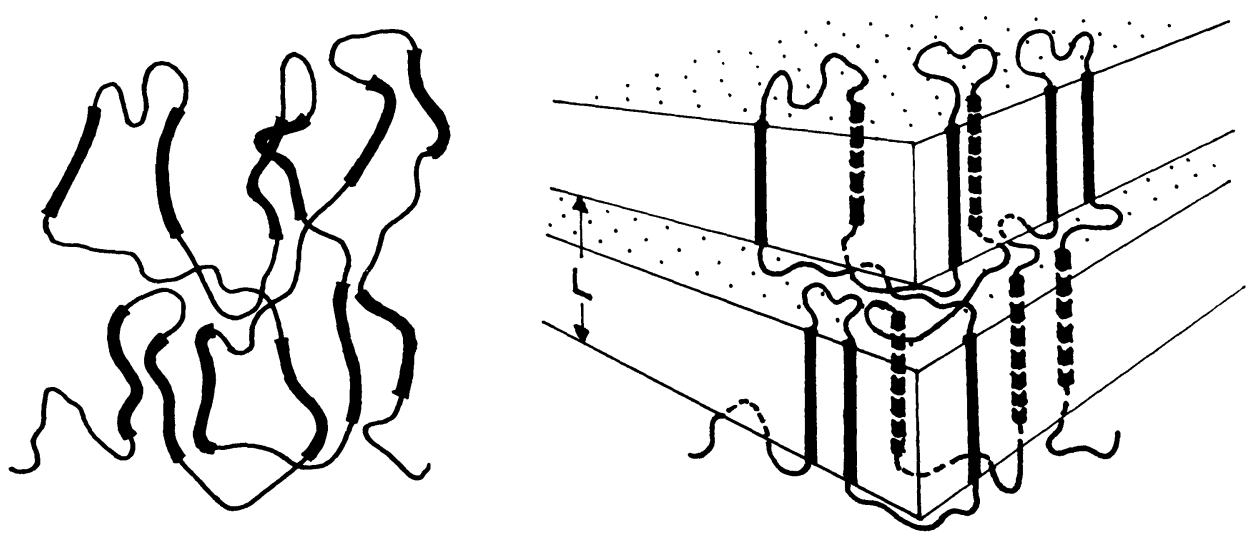

Fig. 15: "Erstarrungsmodel1" (solidification model) of the crystallization process of chain molecules. The fully drawn sequences of the coil are incorporated in the growing lamellae without long range diffusional motion. 
In contrast to the behaviour at small values of $Q$, rather pronounced changes of the scattering curves in the submolecular range can be caused by crystallization. As an example, Fig. 16 shows the comparison of scattering curves of molten and crystallized poly (ethylene oxide) (26). A similar shape of the kratky - plot was found in the case of polyethylene crystallized by quenching (25), whereas in the case of isotactic polypropylene (27) a horizontal plateau was observed.

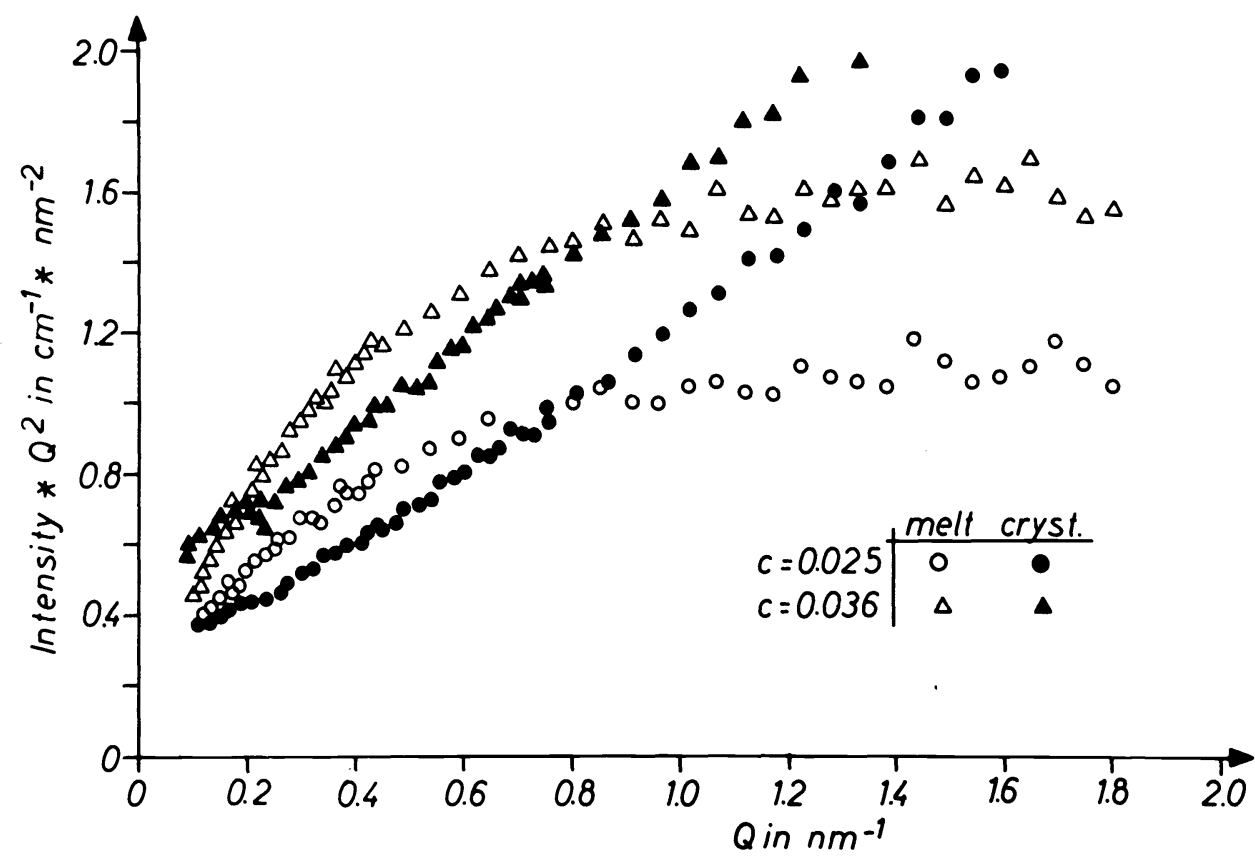

Fig. 16: Comparison of scattering curves of molten and crystallized poly(ethylene oxide) (26) in the range $0.02<0<0.2 \AA^{-1} . \quad(d \sigma / d \Omega) \cdot Q^{2}$ is plotted versus $Q$.

For the evaluation of the neutron scattering pattern Yoon and Flory (28) performed MonteCarlo calculations of the scattering functions for various morphologies that differ according to the mode of chain re-entry into the crystalline lamellae. They were able to explain the results of Schelten et al. (25) (polyethylene crystallized by quenching) by means of an irregular re-entry model. From their point of view a structure as pictured in Fig. 15 is consistent with the observed scattering curve.

In order to discuss in at least a semi-quantitative manner the effect of various model parameters on the form factor $P(Q)$ of the molecules analytical expressions for the scattering curves are required. Such a scattering theory can be developed for a simple model of crystallized stems arranged in a plane, which represents the lamella (see Fig. 17). 


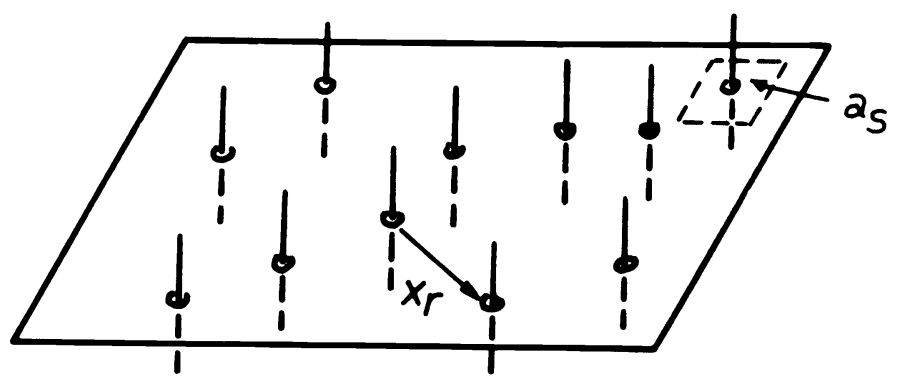

Fig. 17: Simple model of crystallized stems arranged in a lamellar plane.

The overall distribution of the stems is described by

$$
\delta\left(\underline{x}_{r}\right)+\frac{1}{a_{s}} g_{c}\left(\underline{x}_{r}\right) d s
$$

$g_{c}\left(\underline{x}_{r}\right) d s / a_{s}$ is the probability to find a second stem in the area ds at $\underline{x}_{r}$ if there is a stem at $\underline{x}_{r}=0$ ( $a_{s}$ is the average area available for each stem). This model neglects the scattering due to the noncrystallized chain sequences and can be applied only to polymers with high crystallinity. Disregarding very small scattering angles, where the lateral shape of the lamella plays a role, the scattered intensity of the stems can be written as

$$
\frac{d \sigma}{d \Omega}(Q) \sim\left\langle F_{s t}^{2}(Z, R)\left\{1+\frac{2 \pi}{a_{s}} \int_{0}^{\infty}\left[g_{c}\left(x_{r}\right)-1\right] J_{0}\left(R x_{r}\right) x_{r} d x_{r}\right\}\right\rangle
$$

where $Z$ and $R$ are the components of $\underline{Q}$ parallel and perpendicular to the stem direction, respectively. The average has to be taken over all orientations of the lamella, $J_{0}$ is the Bessel function of order zero, and $F_{s t}^{2}$ is the structure factor of the stems.

In a dilute system of uncorrelated stems $\left[g_{c}\left(x_{r}\right)=1\right]$ the Fourier-Bessel - transform in eq. (8) becomes zero and accordingly only the structure factor $F_{s t}^{2}$ of the stems is observed, which in the asymptotic approximation (cross section $\rightarrow 0, Q$ large) turns towards the form factor of a needle:

$$
P(Q)=\frac{\pi}{Q L}-\frac{2}{(Q L)^{2}}
$$

$(L=$ length of the needle). In the Kratky plot this scattering behaviour yields a straight line, the slope of which is essentially given by the scattering cross section per unit length of the stem (see Fig. 18). 


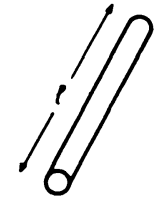

$\Delta B^{s t}=$ diff. in scattering lengths per stem
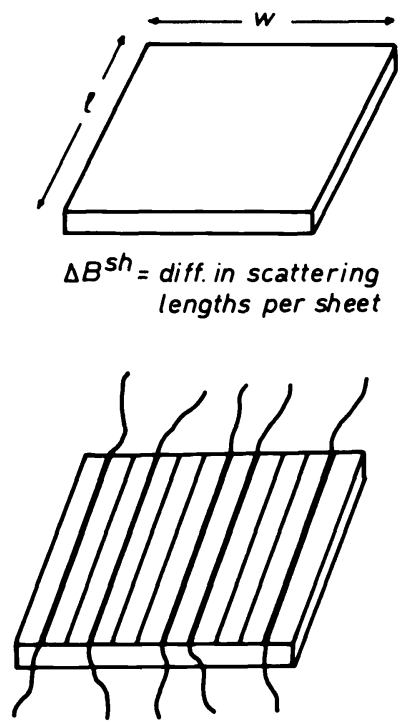
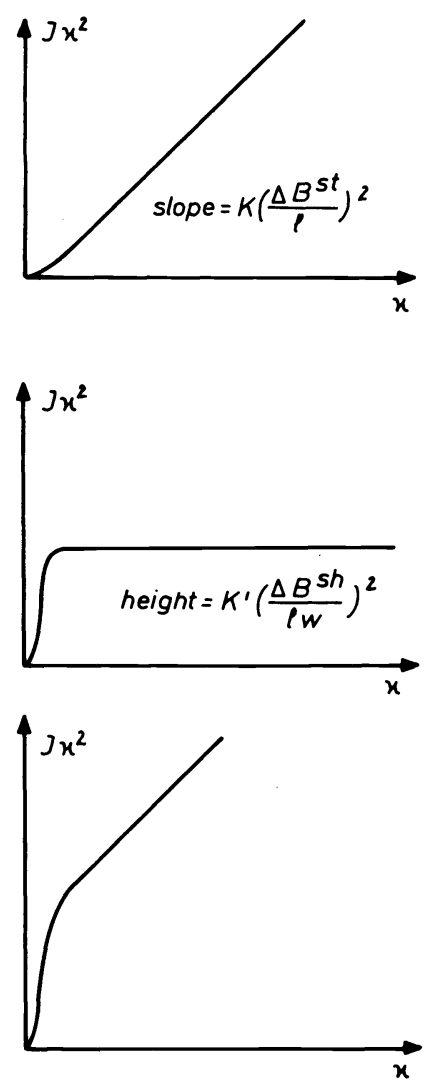

Fig. 18: Schematic drawing showing a possible explanation of the scattering curves of melt crystallized poly(ethylene oxide). The deuterated stems are randomly arranged in the crystallographic net plane of a lamella (two-dimensional switchboard).

Such scattering curves have not been observed up to now. On the other hand it has been found in two cases quenched polyethylene (25) and poly(ethylene oxide) crystallized from a melt (26) that needle scattering occurs in addition to background scattering proportional to $Q^{-2}$, which means that in the Kratky plot $\left[\mathrm{I}(\mathrm{Q}) \mathrm{Q}^{2} \mathrm{vs} . \mathrm{Q}\right]$ the "needle"-straight line is shifted vertically. This scattering behaviour can be explained by various models based on eq. ( 8 ). For example one may assume that the stems are randomly distributed along a crystallographic net plane (see Fig. 18) which means that a two-dimensional switchboard model results, as was demonstrated in Fig. 13. It can be shown (35) that in this case a $Q^{-2}$ background scattering is obtained. Its level is given by the average occupation number of stems in a net plane (see Fig. 18). Other models are also conceivable, however, and so far the only conclusion possible is that the scattering curves are inconsistent with the adjacent-reentry model, as it was pointed out by Yoon and Flory (28).

A reasonable approach for the development of analytical form factors on the basis of eq. (8) is to start with the probability $w\left(x_{r}\right)$ that two consecutive stems along the chain have a distance $x_{r}$. From $w\left(x_{r}\right)$ the pair distribution function $g_{c}\left(x_{r}\right)$ of the stems can be constructed by repeated convolution and the scattering curve can be calculated (35). Figure 19 shows some examples based on a distance distribution wn $\left.\exp \left(-x^{2} / 2<x^{2}\right\rangle\right)$ for consecutive stems. 


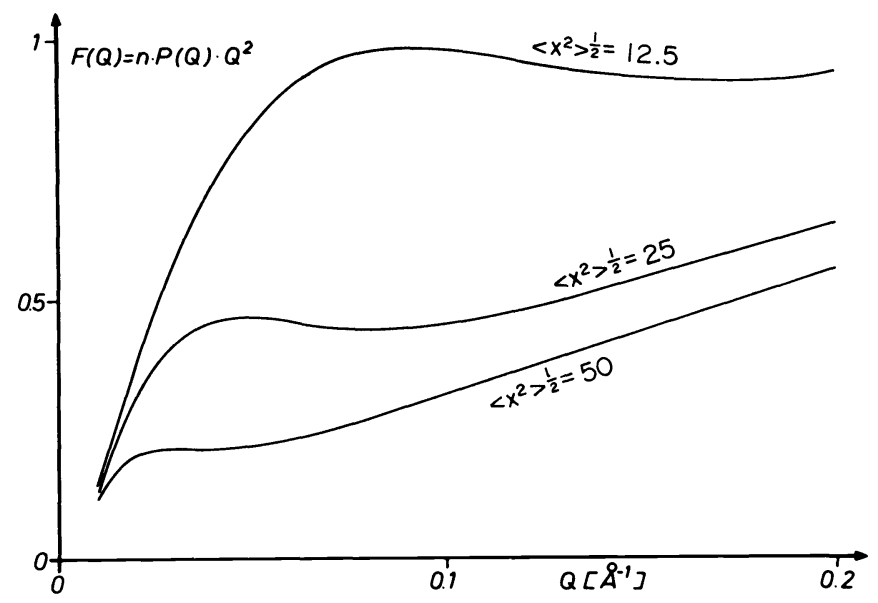

Fig. 19: Kratky - plot of the theoretical scattering curve of crystalline stems in a lamella. Consecutive stem centres have a distance distribution of $w(x) \sim \exp \left(-x^{2} / 2<x^{2}>\right)$.

Presently there are not enough experimental data available in order to ascertain how the configuration of the molecules in the semicrystalline state depends on molecular weight and crystallization conditions. The conclusions drawn so far from neutron scattering experiments are rather controversial and are summarized in Table 4. It is hoped that further improvements in the sample preparation, in the accuracy of scattering measurements and in the analysis of the data will contribute to the solution of the problems inherent in the crystallization of polymers. 
Table 4: Chain configuration in the semicrystalline states as deduced from small angle neutron scattering.

\begin{tabular}{|c|c|c|}
\hline Sample & Conclusions & Ref. \\
\hline $\begin{array}{l}\text { Polyethylene, } \\
\text { rapidly quenched }\end{array}$ & $\begin{array}{l}\text { a) Irreconcilable with adjacent } \\
\text { re-entry } \\
\text { b) Randomly distributed re-entry } \\
\text { with a probability of } 0.7 \\
\text { c) Crystallization in planar sheets } \\
\text { with irregular re-entry }\end{array}$ & $\begin{array}{l}(25) \\
(23) \\
(35)\end{array}$ \\
\hline $\begin{array}{l}\text { Polyethylene, } \\
\text { crystallized under } \\
\text { pressure }\end{array}$ & $\begin{array}{l}\text { rod-like configuration with } \\
\text { rod length equal to lamellar } \\
\text { thickness }(\approx 2000 \AA)\end{array}$ & $(36)$ \\
\hline $\begin{array}{l}\text { Polyethylene, } \\
\text { bulk or crystallized } \\
\text { from solution }\end{array}$ & $\begin{array}{l}\text { Not inconsistent with adjacent } \\
\text { re-entrant folding }\end{array}$ & (37) \\
\hline $\begin{array}{l}\text { Isotactic polypropy- } \\
\text { lene, quenched, or } \\
\text { annealed, or iso- } \\
\text { thermally crystallized }\end{array}$ & $\begin{array}{l}\text { Planar sheets interconnected } \\
\text { by a one-dimensional random walk }\end{array}$ & $(27)$ \\
\hline $\begin{array}{l}\text { Polyethylene oxide, } \\
\text { crystallized by } \\
\text { slow cooling }\end{array}$ & $\begin{array}{l}\text { Crystallization in planar sheets } \\
\text { with irregular re-entry (from } \\
\text { wide angle neutron scattering: } \\
\text { randomly distributed stems) }\end{array}$ & $(26,35)$ \\
\hline
\end{tabular}

\section{Acknowlegement}

The work described here was supported by the Deutsche Forschungsgemeinschaft (Sonderforschungsbereich 41 Mainz/Darmstadt). The author appreciates the help of his colleagues for making available to him their unpublished results. He is especially indebted to Dr. M. Dettenmaier and Dr. B. Ewen for stimulating discussions. Experimental facilities were kindly provided by the Institute Laue-Langevin, Grenoble and the Kernforschungsanlage Jülich. 


\section{References}

1) G.Allen and C.J.Wright, "Neutron Scattering Studies of Polymers" in International Rev. of Science, Vol. 8: Macromolecules Science, Ed. C. E. Bawn, Butterworth, (1975)

2) G.Allen, "A Review of Neutron Scattering with Special Reference to the Measurements of the Unperturbed Dimensions in Macromolecules", J.S. Higgins, "Diffusional Motion of Rubbers", in "Structural Studies of Macromolecules by Spectroscopic Methods", Ed. K.J. Ivin, John Wiley, (1976)

3) J.W. White, "Dynamics of Molecular Crystals, Polymers and Absorbed Species, in: Dynamics of Solids and Liquids by Neutron Scattering", Ed. S.W. Lovesey and T. Springer, Springer Verlag (1977)

4) S.W. Lovesey, in reference 3)

5) W. Marschall and S.W. Lovesey, "Theory of Thermal Neutron Scattering", Oxford, (1971)

6) P.A. Egelstaff, Ed. "Thermal Neutron Scattering", London and New York, (1965)

7) B.T.M. Willis, Ed. "Chemical Applications of Thermal Neutron Scattering", Oxford, (1973)

8) T. Springer, "Quasielastic Neutron Scattering for Investigations of Diffusion Motions in Solids and Liquids", Springer Verlag, Berlin - Heidelberg - New York, (1972)

9) L. van Hove, Phys. Rev. 95, 249, (1954)

10) B. Ewen, D.Richter, accepted for publication in the Journal Chem. Phys. D.Richter, B.Ewen, IAEA Symposium on Neutron Inelastic Scattering, Paper SM-219/35, Wien 1977

11) W. Piesczek, G. Strobl and K. Malzahn, Acta Cryst. B30, 1278, (1974)

G. Strob1, B. Ewen, E.W. Fischer and W. Piesczek, J. Chem. Phys. 61, 5257, (1974)

B. Ewen, E.W. Fischer, W. Piesczek and G. Strobl, J. Chem. Phys. 61, 5265, (1974)

12) H. Fröhlich, Proc. Phys. Soc. London, 54, 422, (1942)

13) H.G. $01 f$ and A. Peterlin, J. Polym. Sci. A2, 8, 771, (1970)

14) B. Alefeld, Kerntechnik 14, 15, (1972)

15) R.L. McCullough, J. Macroml. Sci. Phys. B9, 97, (1974)

16) T. Kitagawa and T. Miyazawa, Adv. Polym. Sci. 9, 335, (1972)

17) K. Kaji, A. Kollmar, B. Ewen, R. Stockmeyer and E.W. Fischer, publication in preparation

18) for references see ref. 21)

19) R.F. Schaufele and T. Shimonouchi, J. Chem. Phys. 47, 3605, (1967)

20) E.W. Fischer, P. Herchenröder, R.St.J. Manley and M. Stamm, to be published in Macromol.

21) P. Herchenröder, E.W. Fischer, G. Lieser, M. Stamm, U.Kalepky and G. Wegner, in prep.

22) R.G. Kirste, W.A. Kruse, J. Schelten, Makromol. Chem. 162, 299, (1972)

J.P. Cotton et a1., Macromolecules 1 , 863, (1974)

H. Benoit, J. Macromol. Sci. B12, 27, (1976)

23) D.Y. Yoon and P.J. Flory, Macromolecules 9 , 294, 299, (1976)

24) G. Lieser, E.W. Fischer and K. Ibel, J. Polym. Sci. B13, 39, (1975) 
25) J. Schelten, B.G. Ballard, G.D. Wignall, G. Longmann and W. Schmatz, Polym. 17, 751, (1976)

26) P. Herchenröder, D. Dettenmaier, E.W. Fischer, G. Wegner and B. Tieke, in preparation

27) D.G.H. Ballard, J. Schelten, P. Cheshire and G.W. Longmann, private communication

28) D.Y. Yoon and P.J. Flory, Polymer 18, 509, (1977)

29) G. Allen, private communication

30) P.J. Flory, J. Amer. Chem. Soc. 84, 2857, (1962)

31) E.W. Fischer, Kolloid Z. U. Z. Polym. 231, 458, (1969)

32) T. Kawai, Makromolekulare Chem. 90, 288, (1966)

33) E.W. Fischer and R. Lorenz, Kolloid Z. U. Z. Polym. 189, 97, (1963)

34) W.M. Ewers, H.G. Zachmann and A. Peterlin, Kolloid Z. U. Z. Polym. 250, 1187, (1972)

35) D. Dettenmaier, E.W. Fischer, P. Herchenröder and M. Stamm, in preparation

36) D.G.H. Ballard, A. Cunningham and J. Schelten, Polym. 18, 259, (1977)

37) D.M. Sadler and A. Keller, Polymer 17, 37, (1976) 